\title{
INTEGRABLE SYSTEMS IN THE PLANE WITH CENTER TYPE LINEAR PART
}

Abstract. We study integrability of two-dimensional autonomous systems in the plane with center type linear part. For quadratic and homogeneous cubic systems we give a simple characterization for integrable cases, and we find explicitly all first integrals for these cases. Finally, two large integrable system classes are determined in the most general nonhomogeneous cases.

1. Introduction. We consider two-dimensional autonomous systems of differential equations of the form

$$
\dot{x}=-y+X(x, y), \quad \dot{y}=x+Y(x, y),
$$

where $X(x, y)$ and $Y(x, y)$ are analytic functions without linear terms defined in a certain neighbourhood of the origin. In the local study of these systems we find three problems closely related to one another: the determination of the origin's stability, existence and number of local limit cycles around the origin and the determination of first integrals when they exist. Poincaré developed an important technique for the general solution of those problems: it consists in finding a formal power series of the form

$$
H(x, y)=\sum_{n=2}^{\infty} H_{n}(x, y),
$$

where $H_{2}(x, y)=\left(x^{2}+y^{2}\right) / 2$ and $H_{n}(x, y)$ are homogeneous polynomials of degree $n$, so that

$$
\dot{H}=\sum_{k=2}^{\infty} V_{2 k}\left(x^{2}+y^{2}\right)^{k},
$$

where $V_{2 k}$ are real numbers called Lyapunov constants. The determination of these constants allows the solution of the three mentioned problems.

1991 Mathematics Subject Classification: Primary 34A05; Secondary 34C05.

Key words and phrases: center-focus problem, integrable systems in the plane. 
In order to solve the problem of stability of the origin, it is sufficient to consider the sign of the first Lyapunov constant different from zero. If it is positive we have asymptotic stability for negative times, and if it is negative we have asymptotic stability for positive times. If all Lyapunov constants are zero, then the origin is stable for all times, but there is no asymptotic stability for any time. In this last case, we have a center at the origin.

The vanishing of all Lyapunov constants is a necessary condition for the integrability of the system (1.1); in this case the series $H(x, y)$ will be a first integral of the system if it were convergent, which is an open question today. On the other hand, it is not always possible to express this first integral (if it exists) by means of elementary functions.

Lyapunov constants are polynomials whose variables are the coefficients of the terms of the development in power series of $X(x, y)$ and $Y(x, y)$. If $X(x, y)$ and $Y(x, y)$ are polynomials of degree $n$, then the ideal generated by $V_{2 k}$ has a finite number of generators by Hilbert's theorem; we let $M(n)$ be the minimum number of generators. It was shown by Shi Songling [7] that under certain hypotheses about Lyapunov generator constants, the number of limit cycles around the origin is at least $M(n)$.

In this work, we study the integrability of the system (1.1) in the case that $X(x, y)$ and $Y(x, y)$ are homogeneous polynomials.

We will call a function $V(x, y)$ a null divergence factor for the system (1.1) if the divergence of the vector field

$$
C=\left(\frac{-y+X(x, y)}{V(x, y)}, \frac{x+Y(x, y)}{V(x, y)}\right)
$$

is zero. In Proposition 1, we show that the existence of a null divergence factor yields the integrability of the system. For quadratic systems (see appendix) and cubic homogeneous systems and for all possible integrability cases there exist null divergence factors which are trigonometric polynomials when expressed in polar coordinates. The main results are as follows:

THEOREM 1. Consider the system

$$
\dot{x}=-y+X_{3}(x, y), \quad \dot{y}=x+Y_{3}(x, y),
$$

where $X_{3}(x, y)$ and $Y_{3}(x, y)$ are homogeneous polynomials of degree 3 . In polar coordinates the system (1.4) becomes

$$
\dot{r}=P_{3}(\varphi) r^{3}, \quad \dot{\varphi}=1+Q_{3}(\varphi) r^{2},
$$

where

$$
\begin{aligned}
& P_{3}(\varphi)=R_{4} \cos \left(4 \varphi+\varphi_{4}\right)+R_{2} \cos \left(2 \varphi+\varphi_{2}\right)+R_{0} \\
& Q_{3}(\varphi)=-R_{4} \sin \left(4 \varphi+\varphi_{4}\right)+\bar{R}_{2} \sin \left(2 \varphi+\bar{\varphi}_{2}\right)+\bar{R}_{0}
\end{aligned}
$$

If the first five Lyapunov constants $V_{4}, V_{6}, V_{8}, V_{10}$ and $V_{12}$ are null, the 
system (1.4) is integrable. There are only the following three cases of integrability:

(i) $R_{0}=0, \bar{\varphi}_{2}=\varphi_{2}$ and $\bar{R}_{2}=-2 R_{2}$. Then $H(r, \varphi)=r^{2} / 2+Q_{3}(\varphi) r^{4} / 4$ is a first integral. In this case, the divergence of the system is zero.

(ii) $R_{0}=0, \bar{\varphi}_{2}=\varphi_{2}$ and $\varphi_{4}=2 \varphi_{2}+\pi / 2$. Then

$$
\begin{aligned}
V(r, \varphi)= & 2 R_{4}+\left(2 R_{4}\left(2 R_{2}+\bar{R}_{2}\right) \sin \left(2 \varphi+\varphi_{2}\right)+R_{2} \bar{R}_{2}\right. \\
& \left.+4 R_{4}\left(\bar{R}_{0}-R_{4}\right)\right) r^{2}+\Delta Q_{3}(\varphi) r^{4}
\end{aligned}
$$

where $\Delta=\left(R_{2}+\bar{R}_{2}\right) R_{2}+2 R_{4}\left(\bar{R}_{0}-R_{4}\right)$, is a null divergence factor. First integrals are the following.

(ii.1) If $\Delta=0$ and $R_{2} \neq 0$ then

(1.8) $H(r, \varphi)=\left(2 R_{4} \sin \left(2 \varphi+\varphi_{2}\right)+\left(R_{2}+\bar{R}_{2}\right)\right) r^{2}$

$$
-\frac{2 R_{4}}{2 R_{2}+\bar{R}_{2}} \ln \left(\left(R_{2}-2 R_{4} \sin \left(2 \varphi+\varphi_{2}\right)\right) r^{2}-\frac{2 R_{4}}{2 R_{2}+\bar{R}_{2}}\right) .
$$

(ii.2) If $\Delta \neq 0$ and $\Delta_{1}=\bar{R}_{2}^{2}+R_{4}\left(R_{4}-\bar{R}_{0}\right)>0$ then

(1.9) $H(r, \varphi)=$

$\frac{\left(\left(2\left(\bar{R}_{0}-R_{4}\right)+\left(\bar{R}_{2}-\sqrt{\Delta_{1}}\right) \sin \left(2 \varphi+\varphi_{2}\right)\right) r^{2}+\frac{\left(\bar{R}_{2}-\sqrt{\Delta_{1}}\right)\left(2 R_{2}+\bar{R}_{2}+\sqrt{\Delta_{1}}\right)}{2 \Delta}\right)^{K_{1}}}{\left(\left(2\left(\bar{R}_{0}-R_{4}\right)+\left(\bar{R}_{2}+\sqrt{\Delta_{1}}\right) \sin \left(2 \varphi+\varphi_{2}\right)\right) r^{2}+\frac{\left(\bar{R}_{2}+\sqrt{\Delta_{1}}\right)\left(2 R_{2}+\bar{R}_{2}-\sqrt{\Delta_{1}}\right)}{2 \Delta}\right)^{K_{2}}}$,

where $K_{1}=2 R_{2}+\bar{R}_{2}+\sqrt{\Delta_{1}}$ and $K_{2}=2 R_{2}+\bar{R}_{2}-\sqrt{\Delta_{1}}$.

(ii.3) If $\Delta \neq 0$ and $\Delta_{1}<0$ then

(1.10) $H(r, \varphi)=$

$$
\begin{aligned}
& \frac{1}{2} \ln \left(\Delta Q_{3}(\varphi) r^{4}+\left(\left(2 \Delta-R_{2}\left(2 R_{2}+\bar{R}_{2}\right)\right)\right.\right. \\
+ & \left.\left.2 R_{4}\left(2 R_{2}+\bar{R}_{2}\right) \sin \left(2 \varphi+\varphi_{2}\right)\right) r^{2}+2 R_{4}\right)+\frac{2 R_{2}+\bar{R}_{2}}{\sqrt{-\Delta_{1}}} \\
\times & \arctan \frac{\Delta\left(2\left(\bar{R}_{0}-R_{4}\right)+\bar{R}_{2} \sin \left(2 \varphi+\varphi_{2}\right)\right) r^{2}+\left(2 \Delta-R_{2}\left(2 R_{2}+\bar{R}_{2}\right)\right)}{\sqrt{-\Delta_{1}}\left(\Delta r^{2} \sin \left(2 \varphi+\varphi_{2}\right)+R_{2}\right)} .
\end{aligned}
$$

(ii.4) If $\Delta \neq 0, \Delta_{1}=0$ and $\bar{R}_{2} \neq 0$, then

$$
\begin{aligned}
H(r, \varphi)= & \ln \left(4 \sqrt{\left(\bar{R}_{0}-R_{4}\right) Q_{3}(\varphi)} r^{2}+\frac{\bar{R}_{2}}{2 R_{2}+\bar{R}_{2}}\right) \\
- & \frac{\frac{4 R_{2}}{2 R_{2}+\bar{R}_{2}}+\left(2 R_{2}+\bar{R}_{2}\right) \sin \left(2 \varphi+\varphi_{2}\right) \cdot r^{2}}{2 \sqrt{\left(\bar{R}_{0}-R_{4}\right) Q_{3}(\varphi)} r^{2}+\frac{\bar{R}_{2}}{2 R_{2}+\bar{R}_{2}}} .
\end{aligned}
$$

(ii.5) If $\Delta \neq 0, \Delta_{1}=0$ and $\bar{R}_{2}=0$, then 
(1.12)

$$
H(r, \varphi)=2 R_{4} \ln \left(\Delta r^{2} \sin \left(2 \varphi+\varphi_{2}\right)+R_{2}\right)+\frac{R_{2}\left(2 R_{4}+\Delta r^{2}\right)}{R_{2}+\Delta r^{2} \sin \left(2 \varphi+\varphi_{2}\right)}
$$

(iii) $R_{0}=0, \bar{\varphi}_{2}=\varphi_{2}, \bar{R}_{0}=0, R_{2}=2 \bar{R}_{2}$ and $\left|R_{4}\right|=\left|\bar{R}_{2}\right|$. Set

$$
\begin{aligned}
W_{4}(r, \varphi)= & +2 R_{4}\left(\sin \left(\varphi_{4}-2 \varphi_{2}\right)+\sin \left(2 \varphi+\varphi_{2}\right)\right) r^{2} \\
& +4 R_{4}^{2} \sin ^{4}\left(\varphi+\frac{\varphi_{4}-\varphi_{2}}{2}\right) r^{4} \quad \text { if } R_{4}=\bar{R}_{2}, \\
W_{4}(r, \varphi)=1 & +2 R_{4}\left(\sin \left(\varphi_{4}-2 \varphi_{2}\right)-\sin \left(2 \varphi+\varphi_{2}\right)\right) r^{2} \\
& +4 R_{4}^{2} \cos ^{4}\left(\varphi+\frac{\varphi_{4}-\varphi_{2}}{2}\right) r^{4} \quad \text { if } R_{4}=-\bar{R}_{2},
\end{aligned}
$$

$W_{6}(r, \varphi)=\sin \left(\varphi_{4}-2 \varphi_{2}\right)+3 R_{4}\left(1+\sin \left(2 \varphi+\varphi_{2}\right) \sin \left(\varphi_{4}-2 \varphi_{2}\right)\right) r^{2}$

$$
+12 R_{4}^{2} \sin ^{3}\left(\varphi+\frac{\varphi_{4}-\varphi_{2}}{2}\right) \cos \left(\varphi+\frac{3 \varphi_{2}-\varphi_{4}}{2}\right) r^{4}
$$

$$
\begin{aligned}
& +8 R_{4}^{3} \sin ^{6}\left(\varphi+\frac{\varphi_{4}-\varphi_{2}}{2}\right) r^{6} \quad \text { if } R_{4}=\bar{R}_{2}, \\
W_{6}(r, \varphi)= & \sin \left(\varphi_{4}-2 \varphi_{2}\right)+3 R_{4}\left(1-\sin \left(2 \varphi+\varphi_{2}\right) \sin \left(\varphi_{4}-2 \varphi_{2}\right)\right) r^{2} \\
& +12 R_{4}^{2} \cos ^{3}\left(\varphi+\frac{\varphi_{4}-\varphi_{2}}{2}\right) \sin \left(\varphi+\frac{3 \varphi_{2}-\varphi_{4}}{2}\right) r^{4} \\
& +8 R_{4}^{3} \cos ^{6}\left(\varphi+\frac{\varphi_{4}-\varphi_{2}}{2}\right) r^{6} \quad \text { if } R_{4}=-\bar{R}_{2} .
\end{aligned}
$$

Then $V(r, \varphi)=W_{4}(r, \varphi) W_{6}(r, \varphi)$ is a null divergence factor. A first integral is

$$
H(r, \varphi)=\left(W_{6}(r, \varphi)\right)^{2} /\left(W_{4}(r, \varphi)\right)^{3} .
$$

The first five Lyapunov constants have been determined by Lloyd [3] as functions of the coefficients of the system written in cartesian coordinates. The novelty of our Theorem 1 is, first of all, the characterization of the integrable cases by means of polar coordinates, which is simple and generalizable as we will see in the following theorem, and on the other hand, an explicit form of first integrals and null divergence factors for all cases.

TheOREM 2. Consider the system (1.1) written in polar coordinates,

$$
\dot{r}=\sum_{s=2}^{\infty} P_{s}(\varphi) r^{s}, \quad \dot{\varphi}=1+\sum_{s=2}^{\infty} Q_{s}(\varphi) r^{s-1} .
$$

In the following two cases, the system (1.13) is integrable (in the sense of vanishing of all Lyapunov constants):

(i) if

$$
\sum_{s=2}^{\infty}(s+1) P_{s}(\varphi)+Q_{s}^{\prime}(\varphi)=0
$$


(ii) if $P_{s}(\varphi)$ and $Q_{s}(\varphi)$ are of the form

$$
\begin{aligned}
P_{s}(\varphi)= & R_{s+1}^{s} \sin (s+1) \omega+R_{s-1}^{s} \sin (s-1) \omega+\ldots \\
& \ldots+ \begin{cases}R_{1}^{s} \sin \omega & \text { for } s \text { even }, \\
R_{2}^{s} \sin 2 \omega & \text { for } s \text { odd },\end{cases} \\
Q_{s}(\varphi)= & R_{s+1}^{s} \cos (s+1) \omega+\bar{R}_{s-1}^{s} \cos (s-1) \omega+\ldots \\
& \ldots+ \begin{cases}\bar{R}_{1}^{s} \cos \omega & \text { for } s \text { even }, \\
\bar{R}_{2}^{s} \cos 2 \omega+\bar{R}_{0}^{s} & \text { for } s \text { odd },\end{cases}
\end{aligned}
$$

where $\omega=\varphi+\varphi_{0}$ with $\varphi_{0}$ arbitrary, and the coefficients $R_{j}^{s}$ and $\bar{R}_{j}^{s}$ are undetermined.

Theorem 2 is the extension of cases (i) and (ii) for quadratic systems (see appendix) and of cubic homogeneous systems to the most general case, independently of the homogeneity of $X(x, y)$ and $Y(x, y)$. Systems corresponding to (i) have null divergence, and those corresponding to (ii) have a certain resonance at the angles.

2. Expression in polar coordinates. From now on, we assume that the nonlinear parts $X(x, y)$ and $Y(x, y)$ of the system (1.1) are given by

$$
X(x, y)=\sum_{s=2}^{\infty} X_{s}(x, y), \quad Y(x, y)=\sum_{s=2}^{\infty} Y_{s}(x, y)
$$

where $X_{s}(x, y)$ and $Y_{s}(x, y)$ are homogeneous polynomials of degree $s$, with $s \geq 2$, that is,

$$
X_{s}(x, y)=\sum_{k=0}^{s} a_{k}^{s} x^{k} y^{s-k}, \quad Y_{s}(x, y)=\sum_{k=0}^{s} b_{k}^{s} x^{k} y^{s-k},
$$

where $a_{k}^{s}$ and $b_{k}^{s}, k=0, \ldots, s$, are arbitrary coefficients.

LEMMA 2.1. In polar coordinates $(r, \varphi)$, we can write the system (1.1) as

$$
\dot{r}=\sum_{s=2}^{\infty} P_{s}(\varphi) r^{s}, \quad \dot{\varphi}=1+\sum_{s=2}^{\infty} Q_{s}(\varphi) r^{s-1},
$$

where $P_{s}(\varphi)$ and $Q_{s}(\varphi), s=2,3, \ldots$, are trigonometric polynomials of the form

$$
\begin{aligned}
P_{s}(\varphi)= & R_{s+1}^{s} \cos \left((s+1) \varphi+\varphi_{s+1}^{s}\right) \\
& +R_{s-1}^{s} \cos \left((s-1) \varphi+\varphi_{s-1}^{s}\right)+\ldots+ \begin{cases}R_{1}^{s} \cos \left(\varphi+\varphi_{1}^{s}\right) & \text { for s even }, \\
R_{0}^{s} & \text { for s odd },\end{cases} \\
Q_{s}(\varphi)= & -R_{s+1}^{s} \sin \left((s+1) \varphi+\varphi_{s+1}^{s}\right) \\
& +\bar{R}_{s-1}^{s} \sin \left((s-1) \varphi+\bar{\varphi}_{s-1}^{s}\right)+\ldots+ \begin{cases}\bar{R}_{1}^{s} \sin \left(\varphi+\bar{\varphi}_{1}^{s}\right) & \text { for s even }, \\
\bar{R}_{0}^{s} & \text { for s odd. }\end{cases}
\end{aligned}
$$


Pr o of. Differentiating $x=r \cos \varphi$ and $y=r \sin \varphi$ with respect to $t$ gives

$$
\dot{r}=\dot{x} \cos \varphi+\dot{y} \sin \varphi, \quad \dot{\varphi}=\frac{\dot{y} \cos \varphi-\dot{x} \sin \varphi}{r},
$$

and from (1.1) and (2.1) we obtain

$$
\begin{aligned}
\dot{r}= & \left(-r \sin \varphi+\sum_{s=2}^{\infty} X_{s}(r \cos \varphi, r \sin \varphi)\right) \cos \varphi \\
& +\left(r \cos \varphi+\sum_{s=2}^{\infty} Y_{s}(r \cos \varphi, r \sin \varphi)\right) \sin \varphi, \\
\dot{\varphi}= & r^{-1}\left(-r \cos \varphi+\sum_{s=2}^{\infty} Y_{s}(r \cos \varphi, r \sin \varphi)\right) \cos \varphi \\
& -r^{-1}\left(r \sin \varphi+\sum_{s=2}^{\infty} X_{s}(r \cos \varphi, r \sin \varphi)\right) \sin \varphi .
\end{aligned}
$$

By homogeneity of $X_{s}$ and $Y_{s}$ and grouping terms, we obtain

$$
\begin{aligned}
& \dot{r}=\sum_{s=2}^{\infty}\left[X_{s}(\cos \varphi, \sin \varphi) \cos \varphi+Y_{s}(\cos \varphi, \sin \varphi) \sin \varphi\right] r^{s}, \\
& \dot{\varphi}=1+\sum_{s=2}^{\infty}\left[Y_{s}(\cos \varphi, \sin \varphi) \cos \varphi-X_{s}(\cos \varphi, \sin \varphi) \sin \varphi\right] r^{s-1},
\end{aligned}
$$

that is, we get (2.3), where, by (2.2),

$$
\begin{aligned}
& P_{s}(\varphi)=\sum_{k=0}^{s} a_{k}^{s} \cos ^{k+1} \varphi \sin ^{s-k} \varphi+\sum_{k=0}^{s} b_{k}^{s} \cos ^{k} \varphi \sin ^{(s+1)-k} \varphi \\
& Q_{s}(\varphi)=\sum_{k=0}^{s} b_{k}^{s} \cos ^{k+1} \varphi \sin ^{s-k} \varphi-\sum_{k=0}^{s} a_{k}^{s} \cos ^{k} \varphi \sin ^{(s+1)-k} \varphi,
\end{aligned}
$$

or

$$
\begin{aligned}
P_{s}(\varphi) & =\sum_{k=0}^{s+1} A_{k}^{s} \cos ^{k} \varphi \sin ^{(s+1)-k} \varphi, \\
Q_{s}(\varphi) & =\sum_{k=0}^{s+1} B_{k}^{s} \cos ^{k} \varphi \sin ^{(s+1)-k} \varphi,
\end{aligned}
$$

where

$$
\begin{array}{llll}
A_{0}^{s}=b_{0}^{s}, & A_{k}^{s}=b_{k}^{s}+a_{k-1}^{s}, & k=1, \ldots, s, & A_{s+1}^{s}=a_{s}^{s}, \\
B_{0}^{s}=-a_{0}^{s}, & B_{k}^{s}=b_{k-1}^{s}-a_{k}^{s}, & k=1, \ldots, s, & B_{s+1}^{s}=b_{s}^{s} .
\end{array}
$$

If $(s+1)-k$ is even, we can write

$\cos ^{k} \varphi \sin ^{(s+1)-k} \varphi=(-1)^{((s+1)-k) / 2} 2^{-s}$ 


$$
\times\left(\cos (s+1) \varphi+\alpha_{s-1}^{k} \cos (s-1) \varphi+\ldots+\left\{\begin{array}{ll}
\alpha_{1}^{k} \cos \varphi & \text { for } s+1 \text { odd } \\
\alpha_{0}^{k} & \text { for } s+1 \text { even }
\end{array}\right)\right.
$$

and if $(s+1)-k$ is odd, then we have

$$
\begin{aligned}
& \cos ^{k} \varphi \sin ^{(s+1)-k} \varphi=(-1)^{(s-k) / 2} 2^{-s} \\
& \times\left(\sin (s+1) \varphi+\alpha_{s-1}^{k} \sin (s-1) \varphi+\ldots+\left\{\begin{array}{ll}
\alpha_{1}^{k} \sin \varphi & \text { for } s+1 \text { odd } \\
\alpha_{0}^{k} & \text { for } s+1 \text { even }
\end{array}\right),\right.
\end{aligned}
$$

where $k=0, \ldots, s+1$ and $\alpha_{i}^{j}$ are integers. Putting these values in (2.4) and grouping terms, we have

$$
\begin{aligned}
& P_{s}(\varphi)=\left(\beta_{s+1}^{s} \cos (s+1) \varphi+\bar{\beta}_{s+1}^{s} \sin (s+1) \varphi\right) \\
& +\left(\beta_{s-1}^{s} \cos (s-1) \varphi+\bar{\beta}_{s-1}^{s} \sin (s-1) \varphi\right) \\
& +\ldots+ \begin{cases}\beta_{1}^{s} \cos \varphi+\bar{\beta}_{1}^{s} \sin \varphi & \text { if } s+1 \text { is odd, } \\
\beta_{0}^{s} & \text { if } s+1 \text { is even, }\end{cases} \\
& Q_{s}(\varphi)=\left(\gamma_{s+1}^{s} \cos (s+1) \varphi+\bar{\gamma}_{s+1}^{s} \sin (s+1) \varphi\right) \\
& +\left(\gamma_{s-1}^{s} \cos (s-1) \varphi+\bar{\gamma}_{s-1}^{s} \sin (s-1) \varphi\right) \\
& +\ldots+ \begin{cases}\gamma_{1}^{s} \cos \varphi+\bar{\gamma}_{1}^{s} \sin \varphi & \text { if } s+1 \text { is odd, } \\
\gamma_{0}^{s} & \text { if } s+1 \text { is even, }\end{cases}
\end{aligned}
$$

with

$$
\begin{gathered}
2 s \beta_{s+1}^{s}=A_{s+1}^{s}-A_{s-1}^{s}+\ldots+ \begin{cases}(-1)^{s / 2} A_{1}^{s} & \text { if } s+1 \text { is odd, } \\
(-1)^{(s+1) / 2} A_{0}^{s} & \text { if } s+1 \text { is even, }\end{cases} \\
2 s \bar{\beta}_{s+1}^{s}=A_{s}^{s}-A_{s-2}^{s}+\ldots+ \begin{cases}(-1)^{s / 2} A_{0}^{s} & \text { if } s+1 \text { is odd, } \\
(-1)^{(s-1) / 2} A_{1}^{s} & \text { if } s+1 \text { is even, }\end{cases} \\
2 s \gamma_{s+1}^{s}=B_{s+1}^{s}-B_{s-1}^{s}+\ldots+ \begin{cases}(-1)^{s / 2} B_{1}^{s} & \text { if } s+1 \text { is odd, } \\
(-1)^{(s+1) / 2} B_{0}^{s} & \text { if } s+1 \text { is even, }\end{cases} \\
2 s \bar{\gamma}_{s+1}^{s}=B_{s}^{s}-A_{s-2}^{s}+\ldots+ \begin{cases}(-1)^{s / 2} B_{1}^{s} & \text { if } s+1 \text { is odd, } \\
(-1)^{(s-1) / 2} B_{0}^{s} & \text { if } s+1 \text { is even, }\end{cases}
\end{gathered}
$$

where $\beta_{j}^{s}, \bar{\beta}_{j}^{s}, j=0, \ldots, s-1$, and $\gamma_{j}^{s}, \bar{\gamma}_{j}^{s}, j=0, \ldots, s-1$, are linear functions of the coefficients $A_{k}^{s}, k=0, \ldots, s+1$, respectively $B_{k}^{s}, k=0, \ldots, s+1$. By (2.5), it is easily shown that in all cases $\beta_{s+1}^{s}=-\bar{\gamma}_{s+1}^{s}$ and $\bar{\beta}_{s+1}^{s}=\gamma_{s+1}^{s}$. On the other hand,

$$
\begin{aligned}
\beta_{k}^{s} \cos k \varphi+\bar{\beta}_{k}^{s} \sin k \varphi & =R_{k}^{s} \cos \left(k \varphi+\varphi_{k}^{s}\right), \\
\gamma_{k}^{s} \cos k \varphi+\bar{\gamma}_{k}^{s} \sin k \varphi & =\bar{R}_{k}^{s} \sin \left(k \varphi+\bar{\varphi}_{k}^{s}\right) ;
\end{aligned}
$$

in particular, if

$$
\beta_{s+1}^{s} \cos (s+1) \varphi+\bar{\beta}_{s+1}^{s} \sin (s+1) \varphi=R_{s+1}^{s} \cos \left((s+1) \varphi+\varphi_{s+1}^{s}\right),
$$

then 


$$
\begin{aligned}
\gamma_{s+1}^{s} \cos (s+1) \varphi+\bar{\gamma}_{s+1}^{s} \sin (s+1) \varphi & =\bar{\beta}_{s+1}^{s} \cos (s+1) \varphi-\beta_{s+1}^{s} \sin (s+1) \varphi \\
& =-R_{s+1}^{s} \sin \left((s+1) \varphi+\varphi_{s+1}^{s}\right)
\end{aligned}
$$

This completes the proof.

3. Homogeneous systems. From now on, we assume that the nonlinear parts, $X(x, y)$ and $Y(x, y)$, of the system (1.1) are homogeneous polynomials of degree $s$, with $s \geq 2$, that is,

$$
\dot{x}=-y+X_{s}(x, y), \quad \dot{y}=x+Y_{s}(x, y),
$$

where

$$
X_{s}(x, y)=\sum_{k=0}^{s} a_{k} x^{k} y^{s-k}, \quad Y_{s}(x, y)=\sum_{k=0}^{s} b_{k} x^{k} y^{s-k},
$$

with $a_{k}$ and $b_{k}, k=0, \ldots, s$, being arbitrary coefficients. By applying Lemma 1 we can write the system (3.1) in polar coordinates as

$$
\dot{r}=P_{s}(\varphi) r^{s}, \quad \dot{\varphi}=1+Q_{s}(\varphi) r^{s-1},
$$

where $P_{s}(\varphi)$ and $Q_{s}(\varphi)$ are trigonometric polynomials of the form

$$
\begin{aligned}
& P_{s}(\varphi)=R_{s+1} \cos \left((s+1) \varphi+\varphi_{s+1}\right) \\
& +R_{s-1} \cos \left((s-1) \varphi+\varphi_{s-1}\right) \\
& +\ldots+ \begin{cases}R_{1} \cos \left(\varphi+\varphi_{1}\right) & \text { if } s \text { is even, } \\
R_{0} & \text { if } s \text { is odd, }\end{cases} \\
& Q_{s}(\varphi)=-R_{s+1} \sin \left((s+1) \varphi+\varphi_{s+1}\right) \\
& +\bar{R}_{s-1} \sin \left((s-1) \varphi+\bar{\varphi}_{s-1}\right) \\
& +\ldots+ \begin{cases}\bar{R}_{1} \sin \left(\varphi+\bar{\varphi}_{1}\right) & \text { if } s \text { is even, } \\
\bar{R}_{0} & \text { if } s \text { is odd. }\end{cases}
\end{aligned}
$$

Proposition 3.1. A Poincaré series for the system $(3.1)$ is $H(r, \varphi)=$ $\sum_{m=0}^{\infty} \bar{H}_{m}(\varphi) r^{m(s-1)+2}$, where $\bar{H}_{0}(\varphi)=1 / 2$ and $\bar{H}_{m}(\varphi), m=0,1, \ldots$, are homogeneous trigonometric polynomials of degree $m(s-1)+2$ satisfying the differential equations

$$
\begin{aligned}
& \frac{d \bar{H}_{m+1}}{d \varphi}+((s-1)+2) \bar{H}_{m}(\varphi) P_{s}(\varphi)+\frac{d \bar{H}_{m}}{d \varphi} Q_{s}(\varphi) \\
&= \begin{cases}0 & \text { if }(m+1)(s-1)+2 \text { is odd }, \\
V_{(m+1)(s-1)+2} & \text { if }(m+1)(s-1)+2 \text { is even },\end{cases}
\end{aligned}
$$

with $V_{(m+1)(s-1)+2}, m=0,1, \ldots$, being the Lyapunov constants.

Proof. In polar coordinates, the series (1.2) takes the form

$$
H(r, \varphi)=\sum_{n=2}^{\infty} H_{n}(\varphi) r^{n}
$$


with $H_{2}(\varphi)=1 / 2$ and $H_{n}(\varphi)$ homogeneous trigonometric polynomials of degree $n$, that is,

$$
H_{n}(\varphi)=\sum_{k=0}^{n} C_{k}^{n} \cos ^{k} \varphi \sin ^{(n+1)-k} \varphi, \quad n=3,4, \ldots
$$

In this case, (1.3) reads

$$
\dot{H}(r, \varphi)=\sum_{k=2}^{\infty} V_{2 k} r^{2 k} .
$$

The evaluation of $\dot{H}(r, \varphi)$ from the system (3.2) yields

$$
\begin{aligned}
\dot{H}(r, \varphi) & =\frac{\partial H}{\partial r} \dot{r}+\frac{\partial H}{\partial \varphi} \dot{\varphi} \\
& =\left(\sum_{n=2}^{\infty} n H_{n}(\varphi) r^{n-1}\right) r^{s} P_{s}(\varphi)+\left(\sum_{n=2}^{\infty} H_{n}^{\prime}(\varphi) r^{n}\right)\left(1+r^{s-1} Q_{s}(\varphi)\right),
\end{aligned}
$$

where the prime stands for $\varphi$-derivative. If we group the powers of $r$ we find

$$
\begin{aligned}
\dot{H}(r, \varphi)= & \sum_{n=2}^{s} H_{n}^{\prime}(\varphi) r^{n} \\
& +\sum_{n=2}^{\infty}\left(H_{n+s-1}^{\prime}(\varphi)+n H_{n}(\varphi) P_{s}(\varphi)+H_{n}^{\prime}(\varphi) Q_{s}(\varphi)\right) r^{n+s-1} .
\end{aligned}
$$

By (3.5), we obtain

$$
\begin{aligned}
\sum_{n=2}^{s} H_{n}^{\prime}(\varphi) r^{n}+\sum_{n=2}^{\infty}\left(H_{n+s-1}^{\prime}(\varphi)\right. & +n H_{n}(\varphi) P_{s}(\varphi) \\
& \left.+H_{n}^{\prime}(\varphi) Q_{s}(\varphi)\right) r^{n+s-1}=\sum_{k=2}^{\infty} V_{2 k} r^{2 k}
\end{aligned}
$$

Equating the coefficients of the powers of $r$, we find in particular for $n=$ $2, \ldots, s$,

$$
H_{n}^{\prime}(\varphi)= \begin{cases}0 & \text { if } n \text { is odd, } \\ V_{n} & \text { if } n \text { is even. }\end{cases}
$$

But since the $H_{n}(\varphi)$ are trigonometric polynomials, $V_{2 k}$ have to be null for $0 \leq 2 k \leq s$, so $d H_{n} / d \varphi=0$ for $n=2, \ldots, s$ and $V_{2 k}=0$ for $0 \leq 2 k \leq s$. If we integrate the polynomials $H_{n}(\varphi)$ with respect to $\varphi$ and take into account their homogeneity, then we get

$$
H_{n}(\varphi)=\left\{\begin{array}{ll}
0 & \text { if } n \text { is odd }, \\
C_{n} & \text { if } n \text { is even, }
\end{array} \quad n=2, \ldots, s,\right.
$$


where the $C_{n}$ are constants (in particular, $C_{2}=1 / 2$ ). For the other terms of (3.6) we have the differential equations

$$
\begin{aligned}
H_{n+s-1}^{\prime}(\varphi)+ & n H_{n}(\varphi) P_{s}(\varphi)+H_{n}^{\prime}(\varphi) Q_{s}(\varphi) \\
\quad & \left\{\begin{array}{ll}
0 & \text { if } n+s-1 \text { is odd, } \\
V_{n+s-1} & \text { if } n+s-1 \text { is even, }
\end{array} \quad n=2,3, \ldots,\right.
\end{aligned}
$$

where initial conditions are given in (3.7). These equations can be considered as a system of $s-1$ independent differential equations: take $n=m(s-1)+k$, $k=2, \ldots, s$ in (3.8) and separate the equations and the initial conditions following the values of $k$, that is,

$$
\begin{array}{r}
H_{(m+1)(s-1)+k}^{\prime}(\varphi)+(m(s-1)+k) H_{m(s-1)+k}(\varphi) P_{s}(\varphi)+H_{m(s-1)+k}^{\prime}(\varphi) Q_{s}(\varphi) \\
=\bar{V}_{m+1}= \begin{cases}0 & \text { if }(m+1)(s-1)+k \text { is odd }, \\
V_{(m+1)(s-1)+k} & \text { if }(m+1)(s-1)+k \text { is even, }\end{cases}
\end{array}
$$

where $k=2, \ldots, s, m=0,1, \ldots$, with

$$
H_{k}(\varphi)=\left\{\begin{array}{ll}
0 & \text { if } k \text { is odd }, \\
C_{k} & \text { if } k \text { is even, }
\end{array} \quad k=2, \ldots, s .\right.
$$

It is obvious that all equations of (3.9) are equal, and only the initial conditions change (which are constant functions). Thus, for each of the equations (3.9) the Lyapunov constants $V_{k}$ that we will find will be the same up to constant multiplicative factors. We can assume, without any loss of generality, that the functions $H_{m(s-1)+k}(\varphi)$ are zero for $k=3, \ldots, s-1$; if we denote $H_{m(s-1)+2}(\varphi)$ by $\bar{H}_{m}(\varphi)$, the system (3.9) will take the form (3.3) with $\bar{H}_{m}(\varphi)$ homogeneous trigonometric polynomials of degree $m(s-1)+2$.

4. Null divergence factors. We call a function $V(x, y)$ a null divergence factor for the system (1.1) if the divergence of the vector field

$$
C=\left(\frac{-y+X(x, y)}{V(x, y)}, \frac{x+Y(x, y)}{V(x, y)}\right)
$$

is null, that is, if

$$
\frac{\partial}{\partial x}\left(\frac{-y+X(x, y)}{V(x, y)}\right)+\frac{\partial}{\partial y}\left(\frac{x+Y(x, y)}{V(x, y)}\right)=0 .
$$

If the system (1.1) is written in polar coordinates (see (2.3)), then the function $V(r, \varphi)$ will be a null divergence factor if

$$
\frac{1}{r} \frac{\partial}{\partial r}\left(\frac{\sum_{s=2}^{\infty} P_{s}(\varphi) r^{s+1}}{V(r, \varphi)}\right)+\frac{\partial}{\partial \varphi}\left(\frac{1+\sum_{s=2}^{\infty} Q_{s}(\varphi) r^{s-1}}{V(r, \varphi)}\right)=0 .
$$


Proposition 4.1. If the system (2.3) has a null divergence factor $V(r, \varphi)$ then the system is integrable, and its first integral is given by

$$
H(r, \varphi)=\int \frac{r\left(1+\sum_{s=2}^{\infty} Q_{s}(\varphi) r^{s-1}\right)}{V(r, \varphi)} d r+\int \frac{\sum_{s=2}^{\infty} P_{s}(\varphi) r^{s+1}}{V(r, \varphi)} d \varphi
$$

Pr o of. The differential equation for the trajectories of the system (2.3) is

$$
\left(1+\sum_{s=2}^{\infty} Q_{s}(\varphi) r^{s-1}\right) d r-\sum_{s=2}^{\infty} P_{s}(\varphi) r^{s} d \varphi=0 .
$$

If $V(r, \varphi)$ is a null divergence factor, then $r / V(r, \varphi)$ is an integrating factor for (4.3) since, by (4.1), we have

$$
\begin{aligned}
\frac{\partial}{\partial \varphi}( & \left.\frac{r\left(1+\sum_{s=2}^{\infty} Q_{s}(\varphi) r^{s-1}\right)}{V(r, \varphi)}\right)+\frac{\partial}{\partial r}\left(\frac{\sum_{s=2}^{\infty} P_{s}(\varphi) r^{s+1}}{V(r, \varphi)}\right) \\
\quad=r \frac{\partial}{\partial \varphi}\left(\frac{1+\sum_{s=2}^{\infty} Q_{s}(\varphi) r^{s-1}}{V(r, \varphi)}\right)+\frac{\partial}{\partial r}\left(\frac{\sum_{s=2}^{\infty} P_{s}(\varphi) r^{s+1}}{V(r, \varphi)}\right) & =0 .
\end{aligned}
$$

Thus the solution of (4.3) is given by the integral (4.2).

In the particular case that the system (2.3) is homogeneous (see (3.2)), the condition (4.1) becomes

$$
\begin{aligned}
& \text { (4.4) } \frac{1}{r} \frac{\partial}{\partial r}\left(\frac{P_{s}(\varphi) r^{s+1}}{V(r, \varphi)}\right)+\frac{\partial}{\partial \varphi}\left(\frac{1+Q_{s}(\varphi) r^{s-1}}{V(r, \varphi)}\right) \\
& =\frac{\left((s+1) P_{s}(\varphi)+Q_{s}^{\prime}(\varphi)\right) r^{s-1}}{V(r, \varphi)}-\frac{P_{s}(\varphi) r^{s}}{(V(r, \varphi))^{2}} \frac{\partial V}{\partial r}-\frac{\left(1+Q_{s}(\varphi) r^{s-1}\right)}{(V(r, \varphi))^{2}} \frac{\partial V}{\partial \varphi}=0 .
\end{aligned}
$$

Corollary 4.2. The function

$$
V(r, \varphi)=\left(1+V_{1}(\varphi) r^{s-1}+V_{2}(\varphi) r^{2(s-1)}+\ldots+V_{p}(\varphi) r^{p(s-1)}\right)^{\alpha}
$$

where $V_{k}(\varphi)$ are trigonometric polynomials of degree $k(s-1)$ and $\alpha$ is a real number, is a null divergence factor for the system (3.2) if the $V_{k}(\varphi)$, $k=1, \ldots, p$, satisfy the system

$$
\begin{aligned}
& \alpha V_{1}^{\prime}-(s+1) P_{s}-Q_{s}^{\prime}=0, \\
& V_{2}^{\prime}+V_{1}^{\prime} Q_{s}+(s-1) V_{1} P_{s}=V_{1} V_{1}^{\prime}, \\
& V_{3}^{\prime}+V_{2}^{\prime} Q_{s}+2(s-1) V_{2} P_{s}=V_{2} V_{1}^{\prime}, \\
& \ldots \ldots \ldots \ldots \ldots \ldots \ldots \ldots \ldots \ldots \ldots \\
& V_{p}^{\prime}+V_{p-1}^{\prime} Q_{s}+(p-1)(s-1) V_{p-1} P_{s}=V_{p-1} V_{1}^{\prime}, \\
& V_{p}^{\prime} Q_{s}+p(s-1) V_{p} P_{s}=V_{p} V_{1}^{\prime} .
\end{aligned}
$$


In this case, a first integral is given by

$$
\begin{aligned}
H(r, \varphi)= & \int \frac{r\left(1+Q_{s} r^{s-1}\right)}{\left(1+V_{1} r^{s-1}+\ldots+V_{p} r^{p(s-1)}\right)^{\alpha}} d r \\
& +\int \frac{P_{s} r^{s+1}}{\left(1+V_{1} r^{s-1}+\ldots+V_{p} r^{p(s-1)}\right)^{\alpha}} d \varphi .
\end{aligned}
$$

Proof. It is sufficient to substitute (4.5) in (4.4) and develop with respect to the powers of $r$.

\section{Cubic systems}

Proof of Theorem 1. A computer calculation of the first five Lyapunov constants gives

$$
\begin{aligned}
V_{4}= & R_{0} \\
2 V_{6}= & R_{2} \bar{R}_{2} \sin \left(\varphi_{2}-\bar{\varphi}_{2}\right) \\
8 V_{8}= & \left(2 R_{2}^{2} \cos \left(2 \varphi_{2}-\varphi_{4}\right)-3 R_{2} \bar{R}_{2} \cos \left(\varphi_{2}+\bar{\varphi}_{2}-\varphi_{4}\right)\right. \\
& \left.-2 \bar{R}_{2}^{2} \cos \left(2 \bar{\varphi}_{2}-\varphi_{4}\right)\right) R_{4}-\left(10 R_{4}^{2}+24 R_{2}^{2}\right) R_{0} \\
& +\left(8 R_{0} \cos \left(\bar{\varphi}_{2}-\varphi_{2}\right)+4 \bar{R}_{0} \sin \left(\bar{\varphi}_{2}-\varphi_{2}\right)\right) R_{2} \bar{R}_{2} . \\
8 V_{10}= & \left(30 R_{4}^{2}+80 R_{2}^{2}\right) R_{0} \bar{R}_{0} \\
& +\left(-6 R_{2}^{2} \cos \left(2 \varphi_{2}-\varphi_{4}\right)+5 R_{2} \bar{R}_{2} \cos \left(\varphi_{2}+\bar{\varphi}_{2}-\varphi_{4}\right)\right. \\
& \left.+4 \bar{R}_{2}^{2} \cos \left(2 \bar{\varphi}_{2}-\varphi_{4}\right)\right) R_{4} \bar{R}_{0} \\
& +\left(26 R_{2}^{2} \sin \left(2 \varphi_{2}-\varphi_{4}\right)+60 R_{2} \bar{R}_{2} \sin \left(\varphi_{2}+\bar{\varphi}_{2}-\varphi_{4}\right)\right. \\
& \left.-8 \bar{R}_{2}^{2} \sin \left(2 \bar{\varphi}_{2}-\varphi_{4}\right)\right) R_{4} R_{0} \\
& +\left(17 R_{2}^{2}-\bar{R}_{2}^{2}+24 R_{0}^{2}-4 \bar{R}_{0}^{2}\right) R_{2} \bar{R}_{2} \sin \left(\bar{\varphi}_{2}-\varphi_{2}\right) \\
& -20 R_{0} \bar{R}_{0} R_{2} \bar{R}_{2} \cos \left(\bar{\varphi}_{2}-\varphi_{2}\right)-2 R_{2}^{2} \bar{R}_{2}^{2} \sin \left(2 \bar{\varphi}_{2}-2 \varphi_{2}\right) \\
192 V_{12}= & 84 R_{0} R_{4}^{4}+\left(-4 R_{2}^{2} \cos \left(2 \varphi_{2}-\varphi_{4}\right)+38 R_{2} \bar{R}_{2} \cos \left(\varphi_{2}+\bar{\varphi}_{2}-\varphi_{4}\right)\right. \\
& \left.+20 \bar{R}_{2}^{2} \cos \left(2 \bar{\varphi}_{2}-\varphi_{4}\right)\right) R_{4}^{3} \\
& +\left(-576 R_{2}^{2}+1008 R_{0}^{2}-1512 \bar{R}_{0}^{2}-1240 \bar{R}_{2}^{2}\right) R_{0} R_{4}^{2} \\
& -\left(80 R_{0} \cos \left(\bar{\varphi}_{2}-\varphi_{2}\right)+108 \bar{R}_{0} \sin \left(\bar{\varphi}_{2}-\varphi_{2}\right)\right) R_{2} \bar{R}_{2} R_{4}^{2} \\
& +\left(-288 R_{2}^{2}-473 \bar{R}_{2}^{2}\right) \cos \left(2 \varphi_{2}-\varphi_{4}\right) R_{2}^{2} R_{4} \\
& +\left(530 R_{2}^{2}-16 \bar{R}_{2}^{2}\right) \cos \left(\varphi_{2}+\bar{\varphi}_{2}-\varphi_{4}\right) R_{2} \bar{R}_{2} R_{4} \\
& +\left(721 R_{2}^{2}-24 \bar{R}_{2}^{2}\right) \cos \left(2 \bar{\varphi}_{2}-\varphi_{4}\right) \bar{R}_{2}^{2} R_{4} \\
& -\left(66 R_{2}^{2} \cos \left(\varphi_{4}+\bar{\varphi}_{2}-3 \varphi_{2}\right)+60 \bar{R}_{2}^{2} \cos \left(\varphi_{4}+\varphi_{2}-3 \bar{\varphi}_{2}\right)\right) R_{2} \bar{R}_{2} R_{4} \\
& +\left(3936 R_{2}^{2} \cos \left(2 \varphi_{2}-\varphi_{4}\right)-1392 R_{2} \bar{R}_{2} \cos \left(\varphi_{2}+\bar{\varphi}_{2}-\varphi_{4}\right)\right. \\
& \left.+840 \bar{R}_{2}^{2} \cos \left(2 \bar{\varphi}_{2}-\varphi_{4}\right)\right) R_{0}^{2} R_{4} \\
& +\left(-2832 R_{2}^{2} \sin \left(2 \varphi_{2}-\varphi_{4}\right)-6324 R_{2} \bar{R}_{2} \sin \left(\varphi_{2}+\bar{\varphi}_{2}-\varphi_{4}\right)\right. \\
&
\end{aligned}
$$




$$
\begin{aligned}
& \left.+696 \bar{R}_{2}^{2} \sin \left(2 \bar{\varphi}_{2}-\varphi_{4}\right)\right) R_{0} \bar{R}_{0} R_{4} \\
& +\left(288 R_{2}^{2} \cos \left(2 \varphi_{2}-\varphi_{4}\right)-144 R_{2} \bar{R}_{2} \cos \left(\varphi_{2}+\bar{\varphi}_{2}-\varphi_{4}\right)\right. \\
& \left.-144 \bar{R}_{2}^{2} \cos \left(2 \bar{\varphi}_{2}-\varphi_{4}\right)\right) \bar{R}_{0}^{2} R_{4} \\
& +\left(3600 R_{2}^{2}-1212 \bar{R}_{2}^{2}+11520 R_{0}^{2}-4320 \bar{R}_{0}^{2}\right) R_{0} R_{2}^{2} \\
& +\left(408 R_{0} \cos \left(2 \bar{\varphi}_{2}-2 \varphi_{2}\right)+168 \bar{R}_{0} \sin \left(2 \bar{\varphi}_{2}-2 \varphi_{2}\right)\right) R_{2}^{2} \bar{R}_{2}^{2} \\
& +\left(-1200 R_{2}^{2}+180 \bar{R}_{2}^{2}-2304 R_{0}^{2}+864 \bar{R}_{0}^{2}\right) R_{2} \bar{R}_{2} R_{0} \cos \left(\bar{\varphi}_{2}-\varphi_{2}\right) \\
& +\left(-1812 R_{2}^{2}+72 \bar{R}_{2}^{2}-2496 R_{0}^{2}+96 \bar{R}_{0}^{2}\right) R_{2} \bar{R}_{2} \bar{R}_{0} \sin \left(\bar{\varphi}_{2}-\varphi_{2}\right) .
\end{aligned}
$$

From the vanishing of the first two constants $V_{4}$ and $V_{6}$, we get

$$
\begin{aligned}
8 V_{8}= & \left(2 R_{2}^{2} \cos \left(2 \varphi_{2}-\varphi_{4}\right)-3 R_{2} \bar{R}_{2} \cos \left(\varphi_{2}+\bar{\varphi}_{2}-\varphi_{4}\right)\right. \\
& \left.-2 \bar{R}_{2}^{2} \cos \left(2 \bar{\varphi}_{2}-\varphi_{4}\right)\right) R_{4}, \\
8 V_{10}= & \left(-6 R_{2}^{2} \cos \left(2 \varphi_{2}-\varphi_{4}\right)+5 R_{2} \bar{R}_{2} \cos \left(\varphi_{2}+\bar{\varphi}_{2}-\varphi_{4}\right)\right. \\
& \left.+4 \bar{R}_{2}^{2} \cos \left(2 \bar{\varphi}_{2}-\varphi_{4}\right)\right) R_{4} \bar{R}_{0} \\
192 V_{12}= & \left(-4 R_{2}^{2} \cos \left(2 \varphi_{2}-\varphi_{4}\right)+38 R_{2} \bar{R}_{2} \cos \left(\varphi_{2}+\bar{\varphi}_{2}-\varphi_{4}\right)\right. \\
& \left.+20 \bar{R}_{2}^{2} \cos \left(2 \bar{\varphi}_{2}-\varphi_{4}\right)\right) R_{4}^{3} \\
& +\left(-288 R_{2}^{2}-473 \bar{R}_{2}^{2}\right) \cos \left(2 \varphi_{2}-\varphi_{4}\right) R_{2}^{2} R_{4} \\
& +\left(530 R_{2}^{2}-16 \bar{R}_{2}^{2}\right) \cos \left(\varphi_{2}+\bar{\varphi}_{2}-\varphi_{4}\right) R_{2} \bar{R}_{2} R_{4} \\
& +\left(721 R_{2}^{2}-24 \bar{R}_{2}^{2}\right) \cos \left(2 \bar{\varphi}_{2}-\varphi_{4}\right) \bar{R}_{2}^{2} R_{4} \\
& -\left(66 R_{2}^{2} \cos \left(\varphi_{4}+\bar{\varphi}_{2}-3 \varphi_{2}\right)+60 \bar{R}_{2}^{2} \cos \left(\varphi_{4}+\varphi_{2}-3 \bar{\varphi}_{2}\right)\right) R_{2} \bar{R}_{2} R_{4} \\
& +\left(288 R_{2}^{2} \cos \left(2 \varphi_{2}-\varphi_{4}\right)-144 R_{2} \bar{R}_{2} \cos \left(\varphi_{2}+\bar{\varphi}_{2}-\varphi_{4}\right)\right. \\
& \left.-144 \bar{R}_{2}^{2} \cos \left(2 \bar{\varphi}_{2}-\varphi_{4}\right)\right) \bar{R}_{0}^{2} R_{4} .
\end{aligned}
$$

If $V_{6}=0$ we have three alternatives: either $\bar{\varphi}_{2}=\varphi_{2}+k \pi, k \in \mathbb{Z}$, or $R_{2}=0$, or $\bar{R}_{2}=0$. In the first case, if we take $\bar{\varphi}_{2}=\varphi_{2}$ and $R_{2} \bar{R}_{2} \neq 0$ we have

$$
\begin{aligned}
8 V_{8}= & \left(2 R_{2}^{2}-3 R_{2} \bar{R}_{2}-2 \bar{R}_{2}^{2}\right) R_{4} \cos \left(2 \varphi_{2}-\varphi_{4}\right) \\
= & \left(R_{2}-2 \bar{R}_{2}\right)\left(2 R_{2}+\bar{R}_{2}\right) R_{4} \cos \left(2 \varphi_{2}-\varphi_{4}\right), \\
8 V_{10}= & \left(-6 R_{2}^{2}+5 R_{2} \bar{R}_{2}+4 \bar{R}_{2}^{2}\right) R_{4} \bar{R}_{0} \cos \left(2 \varphi_{2}-\varphi_{4}\right) \\
= & \left(-3 R_{2}+4 \bar{R}_{2}\right)\left(2 R_{2}+\bar{R}_{2}\right) R_{4} \bar{R}_{0} \cos \left(2 \varphi_{2}-\varphi_{4}\right), \\
192 V_{12}= & \left(\left(-4 R_{2}^{2}+38 R_{2} \bar{R}_{2}+20 \bar{R}_{2}^{2}\right) R_{4}^{3}\right. \\
& +\left(-288 R_{2}^{4}+464 R_{2}^{3} \bar{R}_{2}+248 R_{2}^{2} \bar{R}_{2}^{2}-76 R_{2} \bar{R}_{2}^{3}-24 \bar{R}_{2}^{4}\right) R_{4} \\
& \left.+\left(288 R_{2}^{2}-144 R_{2} \bar{R}_{2}-144 \bar{R}_{2}^{2}\right) \bar{R}_{0}^{2} R_{4}\right) \cos \left(2 \varphi_{2}-\varphi_{4}\right) \\
= & \left(\left(-2 R_{2}+20 \bar{R}_{2}\right) R_{4}^{2}+\left(-144 R_{2}^{3}+304 R_{2}^{2} \bar{R}_{2}-28 R_{2} \bar{R}_{2}^{2}-24 R_{2}^{3}\right)\right. \\
& \left.+\left(144 R_{2}-144 \bar{R}_{2}\right) \bar{R}_{0}^{2}\right)\left(2 R_{2}+\bar{R}_{2}\right) R_{4} \cos \left(2 \varphi_{2}-\varphi_{4}\right) .
\end{aligned}
$$

From the vanishing of $V_{8}$ we find three possible cases: 

(a) $2 R_{2}+\bar{R}_{2}=0$,
(b) $2 \varphi_{2}-\varphi_{4}=\pi / 2+k \pi, k \in \mathbb{Z}$,
(c) $R_{2}-2 \bar{R}_{2}=0$.

If (a) or (b) holds, then $V_{10}=0$ and $V_{12}=0$. So we have the following cases of integrability:

(i) $R_{0}=0, \bar{\varphi}_{2}=\varphi_{2}$ and $2 R_{2}+\bar{R}_{2}=0$.

(ii) $R_{0}=0, \bar{\varphi}_{2}=\varphi_{2}$ and $\varphi_{4}=2 \varphi_{2}+\pi / 2$.

In case (c) the values of $V_{10}$ and $V_{12}$ are

$$
\begin{aligned}
8 V_{10} & =-10 \bar{R}_{2}^{2} R_{4} \bar{R}_{0} \cos \left(2 \varphi_{2}-\varphi_{4}\right), \\
192 V_{12} & =\left(80 R_{4}^{2}-80 \bar{R}_{2}^{2}\right) \bar{R}_{2}^{2} R_{4} \cos \left(2 \varphi_{2}-\varphi_{4}\right),
\end{aligned}
$$

but since we have supposed $\bar{R}_{2} \neq 0$, we get either $\bar{R}_{0}=0$ and $R_{4}=0$, which is a particular case of (ii), or

(iii) $R_{0}=0, \bar{\varphi}_{2}=\varphi_{2}, \bar{R}_{0}=0, R_{2}=2 \bar{R}_{2}$ and $\left|R_{4}\right|=\left|\bar{R}_{2}\right|$.

If $R_{2}=0$ then $8 V_{8}=-2 \bar{R}_{2}^{2} R_{4} \cos \left(2 \bar{\varphi}_{2}-\varphi_{4}\right)$. The vanishing of $V_{8}$ implies that either $\bar{R}_{2}=0$ and $R_{2}=0$, or $R_{2}=0$ and $\varphi_{4}=2 \bar{\varphi}_{2}+\pi / 2+k \pi, k \in \mathbb{Z}$, or $R_{0}=0, R_{2}=0$ and $R_{4}=0$, which are particular cases of (ii). In a similar way, if $\bar{R}_{2}=0$ we also find particular cases of (ii).

We will find first integrals for the five cases we have exhibited:

Case (i): $R_{0}=0, \bar{\varphi}_{2}=\varphi_{2}$ and $2 R_{2}+\bar{R}_{2}=0$ : In this case, the trigonometric polynomials (1.6) of the system (1.5) are of the form

$$
\begin{aligned}
& P_{3}(\varphi)=R_{4} \cos \left(4 \varphi+\varphi_{4}\right)+R_{2} \cos \left(2 \varphi+\varphi_{2}\right), \\
& Q_{3}(\varphi)=-R_{4} \sin \left(4 \varphi+\varphi_{4}\right)-2 R_{2} \sin \left(2 \varphi+\varphi_{2}\right)+\bar{R}_{0} .
\end{aligned}
$$

In this case $P_{3}(\varphi)=-Q_{3}^{\prime}(\varphi) / 4$, because we can write (1.6) as

$$
\dot{r}=-\frac{Q_{3}^{\prime}(\varphi)}{4} r^{3}, \quad \dot{\varphi}=1+Q_{3}(\varphi) r^{2},
$$

and it is easily proved that

$$
H(r, \varphi)=\left(\frac{1}{2}+Q_{3}(\varphi) \frac{r^{2}}{4}\right) r^{2}
$$

is a first integral. Note that the divergence of $C=\left(P_{3}(\varphi) r^{3}, 1+Q_{3}(\varphi) r^{2}\right)$ is $\left(4 P_{3}(\varphi)+Q_{3}^{\prime}(\varphi)\right) r^{2}$, and its vanishing is equivalent to the conditions of the present case.

Case (ii): $R_{0}=0, \bar{\varphi}_{2}=\varphi_{2}$ and $\varphi_{4}=2 \varphi_{2}+\pi / 2$. In this case, the system (1.5) takes the form

$$
\begin{aligned}
& \dot{r}=\left(R_{4} \cos \left(4 \varphi+2 \varphi_{2}+\pi / 2\right)+R_{2} \cos \left(2 \varphi+\varphi_{2}\right)\right) r^{3}, \\
& \dot{\varphi}=1+\left(-R_{4} \sin \left(4 \varphi+2 \varphi_{2}+\pi / 2\right)+\bar{R}_{2} \sin \left(2 \varphi+\varphi_{2}\right)+\bar{R}_{0}\right) r^{2} .
\end{aligned}
$$


We consider the function

$$
\begin{aligned}
V(r, \varphi)=1 & +V_{2}(\varphi) r^{2}+V_{4}(\varphi) r^{4} \\
= & +\left(\left(2 R_{2}+\bar{R}_{2}\right) \sin \left(2 \varphi+\varphi_{2}\right)+\frac{R_{2} \bar{R}_{2}}{2 R_{4}}+2\left(\bar{R}_{0}-R_{4}\right)\right) r^{2} \\
& +\frac{\Delta}{2 R_{4}} Q_{3}(\varphi) r^{4}
\end{aligned}
$$

where $\Delta=\left(R_{2}+\bar{R}_{2}\right) R_{2}+2 R_{4}\left(\bar{R}_{0}-R_{4}\right)$ and $R_{4} \neq 0$; one can easily prove that the trigonometric polynomials $V_{2}(\varphi)$ and $V_{4}(\varphi)$ of degree 2 and 4 respectively satisfy the system

$$
\begin{gathered}
V_{2}^{\prime}-4 P_{3}-Q_{3}^{\prime}=0, \\
V_{4}^{\prime}+2 P_{3} V_{2}+Q_{3} V_{2}^{\prime}=V_{2} V_{2}^{\prime}, \\
4 P_{3} V_{4}+Q_{3} V_{4}^{\prime}=V_{4} V_{2}^{\prime} .
\end{gathered}
$$

Thus, by Corollary $4.2, V(r, \varphi)$ is a null divergence factor. If $R_{4}=0$ we can explicitly verify that the function

$$
V(r, \varphi)=\left(\bar{R}_{2}+\left(R_{2}+\bar{R}_{2}\right) Q_{3}(\varphi) r^{2}\right) r^{2}
$$

is a null divergence factor. Finally, for all cases, we have the null divergence factor given by (1.7).

For the integration of the system (5.1) we make the change of variable $R=r^{2}, v=r^{2} \sin \left(2 \varphi+\varphi_{2}\right)$ to obtain

$$
\begin{aligned}
\dot{R} & =2 r^{2} \cos \left(2 \varphi+\varphi_{2}\right)\left(R_{2} R-2 R_{4} v\right), \\
\dot{v} & =2 r^{2} \cos \left(2 \varphi+\varphi_{2}\right)\left(1+\left(\bar{R}_{0}-R_{4}\right) R+\left(R_{2}+\bar{R}_{2}\right) v\right) .
\end{aligned}
$$

The differential equation of the trajectories of the system (5.2) is

$$
\frac{d R}{d v}=\frac{R_{2} R-2 R_{4} v}{1+\left(R_{2}+\bar{R}_{2}\right) v+\left(\bar{R}_{0}-R_{4}\right) R},
$$

which is a first order differential equation reducible to a homogeneous equation. We have the following cases for its integration:

Case (ii.1): $\Delta=0$. If $R_{2} \neq 0$ we make the change of variable $z=$ $-2 R_{4} v+R_{2} R, v=v$, and the equation (5.3) becomes

$$
\frac{d z}{d v}=\frac{2 R_{4}\left(2 R_{4}-\left(2 R_{2}+\bar{R}_{2}\right) z\right)}{-2 R_{4}+\left(R_{2}+\bar{R}_{2}\right) z} .
$$

The general solution is given by

$$
\left(R_{2}+\bar{R}_{2}\right) z+2 R_{4}\left(2 R_{2}+\bar{R}_{2}\right) v-\frac{2 R_{4} R_{2}}{2 R_{2}+\bar{R}_{2}} \ln \left(\left(2 R_{2}+\bar{R}_{2}\right) z-2 R_{4}\right)=C,
$$

where $2 R_{2}+\bar{R}_{2} \neq 0$. $\quad\left(2 R_{2}+\bar{R}_{2}=0\right.$ is a particular case of (i). $)$ By undoing the changes of variable made previously, we can write a first integral 
of the system (5.1) in this case in the form (1.8). If $R_{2}=0$ then, as $\Delta=\left(R_{2}+\bar{R}_{2}\right) R_{2}+2 R_{4}\left(\bar{R}_{0}-R_{4}\right)=0$, we have either $R_{2}=0$ and $R_{4}=0$, or $R_{2}=0$ and $\bar{R}_{0}=R_{4}$. In the first case, the equation (5.2) becomes $d R / d v=$ 0 , the solution is given by $R=C$, and its first integral is $H(r, \varphi)=r^{2}$, which is a particular case of (1.8). If $R_{2}=0$ and $\bar{R}_{0}=R_{4}$, then (5.2) becomes

$$
\frac{d R}{d v}=\frac{-2 R_{4} v}{1+\bar{R}_{2} v}
$$

with general solution

$$
\bar{R}_{2}\left(\bar{R}_{2} R+2 R_{4} v\right)-2 R_{4} \ln \left(1+\bar{R}_{2} v\right)=C \quad \text { if } \bar{R}_{2} \neq 0 .
$$

By undoing the changes of variables, we can write a first integral as

$$
H(r, \varphi)=\bar{R}_{2}\left(\bar{R}_{2}+2 R_{4} \sin \left(2 \varphi+\varphi_{2}\right)\right) r^{2}-2 R_{4} \ln \left(1+\bar{R}_{2} r^{2} \sin \left(2 \varphi+\varphi_{2}\right)\right),
$$

which is also a particular case of (1.8). obtain

Now consider $\Delta \neq 0$. In $(5.2)$ we set $w=v+R_{2} / \Delta, z=R+2 R_{4} / \Delta$ to

$$
\frac{d z}{d w}=\frac{R_{2} z-2 R_{4} w}{\left(R_{2}+\bar{R}_{2}\right) w+\left(\bar{R}_{0}-R_{4}\right) z},
$$

which is a first order homogeneous differential equation. By the usual change of variable $p=z / w, w=w$, the equation (5.4) becomes

$$
\frac{d w}{w}=-\frac{\left(R_{2}+\bar{R}_{2}\right)+\left(\bar{R}_{0}-R_{4}\right) p}{2 R_{4}+\bar{R}_{2} p+\left(\bar{R}_{0}-R_{4}\right) p^{2}} d p .
$$

Define $\Delta_{1}=\bar{R}_{2}^{2}+8 R_{4}^{2}-8 \bar{R}_{0} R_{4}$, the denominator discriminant of this equation. The general solution of (5.5) is as follows:

Case (ii.2): $\Delta_{1}>0$. Then

$$
\frac{\left(2\left(\bar{R}_{0}-R_{4}\right) p+\bar{R}_{2}-\sqrt{\Delta_{1}}\right)^{\bar{R}_{2}+2 R_{2}+\sqrt{\Delta_{1}}}}{\left(2\left(\bar{R}_{0}-R_{4}\right) p+\bar{R}_{2}+\sqrt{\Delta_{1}}\right)^{\bar{R}_{2}+2 R_{2}-\sqrt{\Delta_{1}}}} w^{2 \sqrt{\Delta_{1}}}=C .
$$

Case (ii.3): $\Delta_{1}<0$. Then

$\frac{1}{2} \ln \left(2 R_{4}+\bar{R}_{2} p+\left(\bar{R}_{0}-R_{4}\right) p^{2}\right)+\ln w$

$$
+\frac{\bar{R}_{2}+2 R_{2}}{\sqrt{-\Delta_{1}}} \arctan \frac{2\left(\bar{R}_{0}-R_{4}\right) p+\bar{R}_{2}}{\sqrt{-\Delta_{1}}}=C .
$$

Case (ii.4): $\Delta_{1}=0$ and $\bar{R}_{2} \neq 0$. Then

$$
\ln \left(2\left(\bar{R}_{0}-R_{4}\right) p+\bar{R}_{2}\right)+\ln w-\frac{\bar{R}_{2}+2 R_{2}}{2\left(\bar{R}_{0}-R_{4}\right) p+\bar{R}_{2}}=C .
$$


Case (ii.5): $\Delta_{1}=0$ and $\bar{R}_{2}=0$. Then $\Delta_{1}=8 R_{4}\left(R_{4}-\bar{R}_{0}\right)$, and there are two possibilities: $R_{4}=0$, which is a particular case of (ii.4), or $R_{4}=\bar{R}_{0}$, giving $2 R_{4} \ln w+R_{2} p=C$.

By undoing the variable changes, we find respectively the first integrals given in (1.9)-(1.12).

There is also a very degenerate case when the denominator in (5.5) is identically null, that is, $R_{4}=0, \bar{R}_{2}=0$ and $\bar{R}_{0}=R_{4}=0$. In this case, the equation (5.3) reads

with general solution

$$
\frac{d R}{d v}=\frac{R_{2} R}{1+R_{2} v},
$$

$$
\frac{R}{1+R_{2} v}=C .
$$

By undoing the variable changes, we have

$$
H(r, \varphi)=\frac{r^{2}}{1+R_{2} r^{2} \sin \left(2 \varphi+\varphi_{2}\right)},
$$

which is a particular case of (1.12).

Case (iii): $R_{0}=0, \bar{\varphi}_{2}=\varphi_{2}, \bar{R}_{0}=0, R_{2}=2 \bar{R}_{2}$ and $\left|R_{4}\right|=\left|\bar{R}_{2}\right|$. If $R_{4}=\bar{R}_{2}$ the system (1.5) reads

$$
\begin{aligned}
\dot{r} & =R_{4}\left(\cos \left(4 \varphi+\varphi_{4}\right)+2 \cos \left(2 \varphi+\varphi_{2}\right)\right) r^{3}, \\
\dot{\varphi} & =1+R_{4}\left(-\sin \left(4 \varphi+\varphi_{4}\right)+\sin \left(2 \varphi+\varphi_{2}\right)\right) r^{2},
\end{aligned}
$$

and if $R_{4}=-\bar{R}_{2}$ then we have

$$
\begin{aligned}
& \dot{r}=R_{4}\left(\cos \left(4 \varphi+\varphi_{4}\right)-2 \cos \left(2 \varphi+\varphi_{2}\right)\right) r^{3}, \\
& \dot{\varphi}=1+R_{4}\left(-\sin \left(4 \varphi+\varphi_{4}\right)-\sin \left(2 \varphi+\varphi_{2}\right)\right) r^{2} .
\end{aligned}
$$

In this last case, setting $\varphi=\omega+\pi / 2$ turns (5.7) into (5.6). Therefore, we only determine the first integrals of (5.6). We consider the function

$$
\begin{aligned}
W_{4}(r, \varphi)= & 1+V_{2}(\varphi) r^{2}+V_{4}(\varphi) r^{4} \\
= & 1+2 R_{4}\left(\left(\sin \left(2 \varphi+\varphi_{2}\right)+\sin \left(\varphi_{4}-2 \varphi_{2}\right)\right) r^{2}\right. \\
& +4 R_{4}^{2} \sin ^{4}\left(\varphi+\frac{\varphi_{4}-\varphi_{2}}{2}\right) r^{4} .
\end{aligned}
$$

It is easily proved that the trigonometric polynomials $V_{2}(\varphi)$ and $V_{4}(\varphi)$, of degree 2 and 4 respectively, satisfy

$$
\begin{gathered}
\frac{5}{2} V_{2}^{\prime}-4 P_{3}-Q_{3}^{\prime}=0, \\
V_{4}^{\prime}+2 P_{3} V_{2}+Q_{3} V_{2}^{\prime}=V_{2} V_{2}^{\prime}, \\
4 P_{3} V_{4}+Q_{3} V_{4}^{\prime}=V_{4} V_{2}^{\prime} .
\end{gathered}
$$


Then, by Corollary 4.2, the function $\left(W_{2}(r, \varphi)\right)^{5 / 2}$ is a null divergence factor, and a first integral of the system (5.6) is obtained by applying the formula (4.7):

$$
\begin{aligned}
H(r, \varphi)= & \int\left(1+V_{2} r^{2}+V_{4} r^{4}\right)^{-5 / 2}\left(1+Q_{3} r^{2}\right) r d r \\
& -\int\left(1+V_{2} r+V_{4} r^{4}\right)^{-5 / 2} P_{3} r^{4} d \varphi \\
= & \frac{\left(V_{2}-2 Q_{3}\right)+\left(V_{4}-Q_{3} V_{2}\right) r^{2}}{\left.3\left(4 V_{4}-V_{2}^{2}\right)\left(1+V_{2} r^{2}+V_{4} r^{4}\right)\right)^{3 / 2}} \\
& +\frac{\left(8 V_{4}-4 Q_{3} V_{2}\right)\left(2 V_{4} r^{2}+V_{2}\right)}{3\left(4 V_{4}-V_{2}^{2}\right)^{2}\left(1+V_{2} r^{2}+V_{4} r^{4}\right)^{1 / 2}} .
\end{aligned}
$$

By substituting the formulas for $V_{2}(\varphi), V_{4}(\varphi)$ and $Q_{3}(\varphi)$ we get

$$
H(r, \varphi)=\frac{W_{6}(r, \varphi)}{6 \cos ^{2}\left(2 \varphi_{2}-\varphi_{4}\right)\left(W_{4}(r, \varphi)\right)^{3 / 2}},
$$

where

$$
\begin{aligned}
W_{6}(r, \varphi)= & \sin \left(\varphi_{4}-2 \varphi_{2}\right) \\
& +3 R_{4}\left(\sin \left(2 \varphi+\varphi_{2}\right) \sin \left(\varphi_{4}-2 \varphi_{2}\right)+1\right) r^{2} \\
& +12 R_{4}^{2} \sin ^{3}\left(\varphi+\frac{\varphi_{4}-\varphi_{2}}{2}\right) \cos \left(\varphi+\frac{3 \varphi_{2}-\varphi_{4}}{2}\right) r^{4} \\
& +8 R_{4}^{3} \sin ^{6}\left(\varphi+\frac{\varphi_{4}-\varphi_{2}}{2}\right) r^{6}
\end{aligned}
$$

We can assume that $\cos ^{2}\left(\varphi_{4}-2 \varphi_{2}\right) \neq 0$, because the opposite case has been studied in (ii). Finally, we can take as first integral the function

$$
H(r, \varphi)=\frac{W_{6}(r, \varphi)}{\left(W_{4}(r, \varphi)\right)^{3 / 2}} .
$$

By squaring both sides of (5.8), the considered first integrals can be expressed as the quotient of two trigonometric polynomials where the numerator is the square of a squared sixth degree trigonometric polynomial, and the denominator is the cube of a fourth degree polynomial.

Proposition 6.1. The product $W(r, \varphi)=W_{2}(r, \varphi) W_{3}(r, \varphi)$ is a null divergence factor for the system (5.6).

Proof. It is sufficient to show that

$$
\frac{1}{r} \frac{\partial}{\partial r}\left(\frac{P_{3}(\varphi) r^{4}}{W_{4}(r, \varphi) W_{6}(r, \varphi)}\right)+\frac{\partial}{\partial \varphi}\left(\frac{1+Q_{3}(\varphi) r^{2}}{W_{4}(r, \varphi) W_{6}(r, \varphi)}\right)
$$




$$
\begin{aligned}
= & \frac{W_{4} W_{6}\left(4 P_{3}+Q_{3}^{\prime}\right) r^{2}-P_{3} r^{3}\left(W_{4} \frac{\partial W_{6}}{\partial r}+W_{6} \frac{\partial W_{4}}{\partial r}\right)}{\left(W_{4} W_{6}\right)^{2}} \\
& -\frac{\left(1+Q_{3} r^{2}\right)\left(W_{4} \frac{\partial W_{6}}{\partial \varphi}+W_{6} \frac{\partial W_{4}}{\partial \varphi}\right)}{\left(W_{4} W_{6}\right)^{2}}=0 .
\end{aligned}
$$

But $\left(W_{4}(r, \varphi)\right)^{5 / 2}$ is a null divergence factor, so by applying (4.4) we have

$$
W_{4}\left(4 P_{3}+Q_{3}^{\prime}\right) r^{2}-\frac{5}{2}\left(P_{3} r^{3} \frac{\partial W_{4}}{\partial r}-\left(1+Q_{3} r^{2}\right) \frac{\partial W_{4}}{\partial \varphi}\right)=0,
$$

and by differentiating the first integral (5.8) with respect to $t$, we have

$$
\begin{aligned}
& \left(3 W_{6} \frac{\partial W_{4}}{\partial r}-2 W_{4} \frac{\partial W_{6}}{\partial r}\right) P_{3} r^{3} \\
& +\left(3 W_{6} \frac{\partial W_{4}}{\partial \varphi}-2 W_{4} \frac{\partial W_{6}}{\partial \varphi}\right)\left(1+Q_{3} r^{2}\right)=0 .
\end{aligned}
$$

Finally, by multiplying the equation (5.10) by $2 W_{6}$ and adding it to (5.11) we obtain (5.9).

\section{General systems}

Proof of Theorem 2. For type (i) systems, it is easily proved that

$$
H(r, \varphi)=\frac{r^{2}}{2}+\sum_{s=3}^{\infty} Q_{s}(\varphi) \frac{r^{s+1}}{s+1}
$$

is a first integral of the system (1.14).

For type (ii) systems, let

$$
H(r, \varphi)=\sum_{n=2}^{\infty} H_{n}(\varphi) r^{n}, \quad H_{2}(\varphi)=\frac{1}{2},
$$

be the Poincaré series that we already considered before. From (1.15) it follows that

$$
\begin{aligned}
\dot{H}(r, \varphi)= & \frac{\partial H}{\partial r} \dot{r}+\frac{\partial H}{\partial \varphi} \dot{\varphi} \\
= & \left(\sum_{n=2}^{\infty} n H_{n}(\varphi) r^{n-1}\right)\left(\sum_{s=2}^{\infty} P_{s}(\varphi) r^{s}\right) \\
& +\left(\sum_{n=2}^{\infty} H_{n}^{\prime}(\varphi) r^{n}\right)\left(1+\sum_{s=2}^{\infty} Q_{s}(\varphi) r^{s-1}\right) .
\end{aligned}
$$


Grouping powers of $r$ gives

$$
\begin{aligned}
\dot{H}(r, \varphi)=\sum_{n=3}^{\infty}\left(H_{n}^{\prime}(\varphi)+\sum_{k=1}^{n-2}(k+1) H_{k+1}(\varphi) P_{n-k}(\varphi)\right. & \\
& \left.+\sum_{k=1}^{n-2} H_{k+1}^{\prime}(\varphi) Q_{n-k}(\varphi)\right) r^{n}
\end{aligned}
$$

On the other hand,

$$
\dot{H}(r, \varphi)=\sum_{k=2}^{\infty} V_{2 k} r^{2 k}
$$

hence

$$
\begin{array}{r}
H_{n}^{\prime}(\varphi)+\sum_{k=1}^{n-2}(k+1) H_{k+1}(\varphi) P_{n-k}(\varphi)+\sum_{k=1}^{n-2} H_{k+1}^{\prime}(\varphi) Q_{n-k}(\varphi) \\
= \begin{cases}0 & \text { if } n \text { is odd } \\
V_{n} & \text { if } n \text { is even }\end{cases}
\end{array}
$$

where $n=3,4, \ldots$, and $H_{2}(\varphi)=1 / 2$.

Using the trigonometric formulas

$$
\begin{aligned}
\sin m \omega= & \sin \omega\left\{(2 \cos \omega)^{m-1}-\left(\begin{array}{c}
m-2 \\
1
\end{array}\right)(2 \cos \omega)^{m-3}\right. \\
& \left.+\left(\begin{array}{c}
m-3 \\
2
\end{array}\right)(2 \cos \omega)^{m-5}-\ldots\right\} \\
\cos m \omega= & \frac{1}{2}\left\{(2 \cos \omega)^{m}-\frac{m}{1}(2 \cos \omega)^{m-2}+\frac{m}{2}\left(\begin{array}{c}
m-3 \\
1
\end{array}\right)(2 \cos \omega)^{m-4}\right. \\
& \left.-\frac{m}{3}\left(\begin{array}{c}
m-4 \\
2
\end{array}\right)(2 \cos \omega)^{m-6}+\ldots\right\}
\end{aligned}
$$

in (1.15) we have

$$
\begin{aligned}
& P_{s}(\varphi) \\
& \quad=\sin \omega\left(C_{s+1}^{s} \cos ^{s} \omega+C_{s-1}^{s} \cos ^{s-2} \omega+\ldots+\left\{\begin{array}{ll}
C_{1}^{s} \cos \omega & \text { if } s \text { is odd } \\
C_{0}^{s} & \text { if } s \text { is even }
\end{array}\right),\right. \\
& Q_{s}(\varphi) \\
& \quad=C_{s+1}^{s} \cos ^{s+1} \omega+\bar{C}_{s-1}^{s} \cos ^{s-1} \omega+\ldots+ \begin{cases}\bar{C}_{2}^{s} \cos 2 \omega+\bar{C}_{0}^{s} & \text { if } s \text { is odd }, \\
\bar{C}_{1}^{s} \cos \omega & \text { if } s \text { is even, }\end{cases}
\end{aligned}
$$

where $\omega=\varphi+\varphi_{0}, \varphi_{0}$ is arbitrary and the coefficients $C_{j}^{s}, \bar{C}_{j}^{s}$ are linear functions of $R_{j}^{s}, \bar{R}_{j}^{s}$ respectively, $j=0, \ldots, s+1$.

By setting $z=\cos \omega$, we can write $P_{s}(\varphi)=\sin \omega \bar{P}_{s}(z)$ and $Q_{s}(\varphi)=$ $\bar{Q}_{s}(z)$, where

$$
\bar{P}_{s}(z)=C_{s+1}^{s} z^{s}+C_{s-1}^{s} z^{s-2}+\ldots+ \begin{cases}C_{1}^{s} z & \text { if } s \text { is odd } \\ C_{0}^{s} & \text { if } s \text { is even }\end{cases}
$$




$$
\bar{Q}_{s}(z)=C_{s+1}^{s} z^{s+1}+\bar{C}_{s-1}^{s} z^{s-1}+\ldots+ \begin{cases}\bar{C}_{2}^{s} z^{2}+\bar{C}_{0}^{s} & \text { if } s \text { is odd, } \\ \bar{C}_{1}^{s} z & \text { if } s \text { is even, }\end{cases}
$$

which are polynomials in $z$. On the other hand, $d / d \varphi=-\sin \omega d / d z$ and the differential equation (3.3) becomes

$$
\begin{array}{r}
\sin \omega\left(-\frac{d H_{n}}{d z}+\sum_{k=1}^{n-2}(k+1) H_{k+1}(z) \bar{P}_{n-k}(z)-\frac{d H_{k+1}}{d z} \bar{Q}_{n-k}(z)\right) \\
= \begin{cases}0 & \text { if } s \text { is odd } \\
V_{n} & \text { if } s \text { is even }\end{cases}
\end{array}
$$

where $n=3,4, \ldots$, and $H_{2}(z)=1 / 2$. If we set

$H_{n}(z)=$

$$
\sum_{k=1}^{n-2} \int_{0}^{z}\left((k+1) H_{k+1}(\tau) \bar{P}_{n-k}(\tau)-\frac{d H_{k+1}}{d \tau} \bar{Q}_{n-k}(\tau)\right) d \tau, \quad n=3,4, \ldots,
$$

then since $\bar{P}_{n-k}(z)$ and $\bar{Q}_{n-k}(z)$ are polynomials in $z$ of degrees $n-k-1$ and $n-k$ respectively, it is easy to prove by recurrence that $H_{n}(z)$ are polynomials in $z$ of degree $n$ and of the form

$$
H_{n}(z)=A_{n}^{n} z^{n}+A_{n-2}^{n} z^{n-2}+\ldots+ \begin{cases}A_{1}^{n} z & \text { if } n \text { is odd } \\ A_{0}^{n} & \text { if } n \text { is even }\end{cases}
$$

where $n=3,4, \ldots$, and $A_{n}^{k}$ are functions of the coefficients $C_{i}^{j}$ and $\bar{C}_{i}^{j}$, $0 \leq i \leq j+1,2 \leq j \leq n-1$, and by setting $V_{n}$ equal to zero, for $n=3,4, \ldots$, the $H_{n}(\cos \omega)$ are solutions of $(6.2)$. Notice that the polynomials $H_{n}(z)$ are Chebyshev polynomials.

Appendix. Consider the system

$$
\dot{x}=-y+X_{2}(x, y), \quad \dot{y}=x+Y_{2}(x, y),
$$

where $X_{2}(x, y)$ and $Y_{2}(x, y)$ are second degree homogeneous polynomials. In polar coordinates, this system reads

$$
\dot{r}=P_{2}(\varphi) r^{2}, \quad \dot{\varphi}=1+Q_{2}(\varphi) r,
$$

where

$$
\begin{aligned}
& P_{2}(\varphi)=R_{3} \cos \left(3 \varphi+\varphi_{3}\right)+R_{1} \cos \left(\varphi+\varphi_{1}\right), \\
& Q_{2}(\varphi)=-R_{3} \sin \left(3 \varphi+\varphi_{3}\right)+\bar{R}_{1} \sin \left(\varphi+\bar{\varphi}_{1}\right) .
\end{aligned}
$$

If the first three Lyapunov constants $V_{4}, V_{6}$ and $V_{8}$ are null, the system is integrable. There are the following four possible cases of integrability:

(i) $\bar{\varphi}_{1}=\varphi_{1}$ and $\bar{R}_{1}=-3 R_{1}$. Then

$$
H(r, \varphi)=\frac{r^{2}}{2}+Q_{2}(\varphi) \frac{r^{3}}{3}
$$


is a first integral. Note that the divergence of the system is null.

(ii) $\bar{\varphi}_{1}=\varphi_{1}$ and $\varphi_{3}=3 \varphi_{1}$. Then

$$
\begin{aligned}
V(r, \varphi)= & 4 R_{3}+4 R_{3}\left(\left(3 R_{1}+\bar{R}_{1}\right) \sin \left(\varphi+\varphi_{1}\right)\right) r \\
& +\left(R_{1}+R_{3}\right)\left(\left(\bar{R}_{1}-3 R_{3}\right)\left(R_{1}-\bar{R}_{1}+4 R_{3}\right)\right. \\
& \left.+4 R_{3}\left(3 R_{1}+\bar{R}_{1}\right) \sin ^{2}\left(\varphi+\varphi_{1}\right)\right) r^{2} \\
& +\left(R_{1}+R_{3}\right)\left(R_{1}-\bar{R}_{1}+4 R_{3}\right)\left(R_{1}+\bar{R}_{1}-2 R_{3}\right) Q_{2}(\varphi) r^{3}
\end{aligned}
$$

is a null divergence factor. First integrals $H(r, \varphi)$ are the following.

(ii.1) If $R_{3}+R_{1} \neq 0, R_{1}+\bar{R}_{1}-2 R_{3} \neq 0,3 R_{3}-\bar{R}_{1} \neq 0$ and $4 R_{3}+R_{1}-\bar{R}_{1}$ $\neq 0$ then

$$
\begin{aligned}
H(r, \varphi)= & \frac{\left(1+\left(R_{1}+\bar{R}_{1}-2 R_{3}\right) r \sin \left(\varphi+\varphi_{1}\right)\right)^{k}}{D}, \\
D= & \left(4 R_{3}+R_{1}-\bar{R}_{1}\right)\left(4 R_{3} \sin ^{2}\left(\varphi+\varphi_{1}\right)-\left(3 R_{3}-\bar{R}_{1}\right)\right) r^{2} \\
& +8 R_{3} r \sin \left(\varphi+\varphi_{1}\right)+\frac{4 R_{3}}{R_{3}+R_{1}}, \\
k= & \frac{2\left(R_{1}+R_{3}\right)}{R_{1}+\bar{R}_{1}-2 R_{3}} .
\end{aligned}
$$

(ii.2) If $R_{3}+R_{1}=0$ then

$$
\begin{aligned}
H(r, \varphi)= & \left(1-\frac{4 R_{1} \sin ^{2}\left(\varphi+\varphi_{1}\right)}{\left(3 R_{1}+\bar{R}_{1}\right)}\right) r^{2}+\frac{8 R_{1} r \sin \left(\varphi+\varphi_{1}\right)}{\left(3 R_{1}+\bar{R}_{1}\right)^{2}} \\
& -\frac{8 R_{1} \ln \left(1+\left(3 R_{1}+\bar{R}_{1}\right) r \sin \left(\varphi+\varphi_{1}\right)\right)}{\left(3 R_{1}+\bar{R}_{1}\right)^{3}} .
\end{aligned}
$$

(ii.3) If $R_{1}+\bar{R}_{1}-2 R_{3}=0$ and $R_{3}+R_{1} \neq 0$ then

$$
\begin{aligned}
H(r, \varphi)= & \ln \left(\frac{2 R_{3}}{\left(R_{1}+R_{3}\right)^{3}}+\frac{4 R_{3} r \sin \left(\varphi+\varphi_{1}\right)}{\left(R_{1}+R_{3}\right)^{2}}\right. \\
& \left.+\left(\frac{4 R_{3} \sin ^{2}\left(\varphi+\varphi_{1}\right)}{\left(R_{1}+R_{3}\right)}-1\right) r^{2}\right) \\
& -2\left(R_{1}+R_{3}\right) r \sin \left(\varphi+\varphi_{1}\right) .
\end{aligned}
$$

(ii.4) If $3 R_{3}-\bar{R}_{1}=0$ and $R_{3}+R_{1} \neq 0$ then

$$
\begin{aligned}
H(r, \varphi)= & \frac{12 R_{3}+16 R_{3}\left(R_{3}+R_{1}\right) r \sin \left(\varphi+\varphi_{1}\right)+\left(R_{3}+R_{1}\right)^{3} r^{2}}{\left(1+\left(R_{1}+R_{3}\right) r \sin \left(\varphi+\varphi_{1}\right)\right)^{2}} \\
& +8 R_{3} \ln \left(1+\left(R_{3}+R_{1}\right) r \sin \left(\varphi+\varphi_{1}\right)\right) .
\end{aligned}
$$

(ii.5) If $4 R_{3}+R_{1}-\bar{R}_{1}=0$ and $R_{3}+R_{1} \neq 0$ then

$$
H(r, \varphi)=\frac{-R_{3}+2 R_{3}\left(R_{1}+R_{3}\right) r \sin \left(\varphi+\varphi_{1}\right)}{1+2\left(R_{3}+R_{1}\right) r \sin \left(\varphi+\varphi_{1}\right)}
$$




$$
\begin{aligned}
& +\frac{\left(R_{3}+R_{1}\right)^{2}\left(4 R_{3} \sin ^{2}\left(\varphi+\varphi_{1}\right)+\left(R_{1}+R_{3}\right)\right) r^{2}}{1+2\left(R_{3}+R_{1}\right) r \sin \left(\varphi+\varphi_{1}\right)} \\
& -2 R_{3} \ln \left(1+2\left(R_{3}+R_{1}\right) r \sin \left(\varphi+\varphi_{1}\right)\right) .
\end{aligned}
$$

(iii) If $\bar{\varphi}_{1}=\varphi_{1}, R_{1}=3 \bar{R}_{1}$ and $\left|R_{3}\right|=\left|\bar{R}_{1}\right|$, we consider the functions

$W_{2}(r, \varphi)$

$$
= \begin{cases}1+4 R_{3} \sin \left(\varphi+\varphi_{1}\right) r+4 R_{3}^{2} \sin ^{2}\left(\varphi+\frac{\varphi_{3}-\varphi_{1}}{2}\right) r^{2} & \text { if } R_{3}=\bar{R}_{1}, \\ 1-4 R_{3} \sin \left(\varphi+\varphi_{1}\right) r+4 R_{3}^{2} \cos ^{2}\left(\varphi+\frac{\varphi_{3}-\varphi_{1}}{2}\right) r^{2} & \text { if } R_{3}=-\bar{R}_{1},\end{cases}
$$

$W_{3}(r, \varphi)=1+6 R_{3} \sin \left(\varphi+\varphi_{1}\right) r$

$$
\begin{aligned}
& +12 R_{3}^{2} \cos \left(\frac{\varphi_{3}-3 \varphi_{1}}{2}\right) \sin \left(\varphi+\frac{\varphi_{3}-\varphi_{1}}{2}\right) \sin \left(\varphi+\varphi_{1}\right) r^{2} \\
& +8 R_{3}^{3} \cos \left(\frac{\varphi_{3}-3 \varphi_{1}}{2}\right) \sin ^{3}\left(\varphi+\frac{\varphi_{3}-\varphi_{1}}{2}\right) r^{3} \quad \text { if } R_{3}=\bar{R}_{1},
\end{aligned}
$$

$W_{3}(r, \varphi)=1-6 R_{3} \sin \left(\varphi+\varphi_{1}\right) r$

$$
\begin{aligned}
& -12 R_{3}^{2} \sin \left(\frac{\varphi_{3}-3 \varphi_{1}}{2}\right) \cos \left(\varphi+\frac{\varphi_{3}-\varphi_{1}}{2}\right) \sin \left(\varphi+\varphi_{1}\right) r^{2} \\
& +8 R_{3}^{3} \sin \left(\frac{\varphi_{3}-3 \varphi_{1}}{2}\right) \cos ^{3}\left(\varphi+\frac{\varphi_{3}-\varphi_{1}}{2}\right) r^{3} \quad \text { if } R_{3}=-\bar{R}_{1} .
\end{aligned}
$$

Then $V(r, \varphi)=W_{2}(r, \varphi) W_{3}(r, \varphi)$ is a null divergence factor, and $H(r, \varphi)=$ $\left(W_{3}(r, \varphi)\right)^{2} /\left(W_{2}(r, \varphi)\right)^{3}$ is a first integral.

(iv) If $\bar{\varphi}_{1}=\varphi_{1}$ and $R_{1}=\bar{R}_{1}$ then

$$
\begin{aligned}
V(r, \varphi)= & 1+V_{1}(\varphi) r+V_{2}(\varphi) r^{2}+V_{3}(\varphi) r^{3} \\
= & 1+4 R_{1} \sin \left(\varphi+\varphi_{1}\right) r+\left(3\left(R_{1}^{2}-R_{3}^{2}\right)\right. \\
& \left.-2 R_{1}^{2} \cos \left(2 \varphi+2 \varphi_{1}\right)-2 R_{1} R_{3} \cos \left(2 \varphi+\varphi_{3}-\varphi_{1}\right)\right) r^{2} \\
& +2\left(R_{1}^{2}-R_{3}^{2}\right) Q_{2}(\varphi) r^{3}
\end{aligned}
$$

is a null divergence factor. In order to determine first integrals, we consider the decomposition of $Q_{2}(\varphi)$ and $P_{2}(\varphi)$ into real or complex linear factors, that is,

$$
\begin{aligned}
P_{2}(\varphi) & =\lambda \cos \left(\varphi+\omega_{1}\right) \cos \left(\varphi+\omega_{2}\right) \cos \left(\varphi+\omega_{3}\right), \\
Q_{2}(\varphi) & =\lambda \sin \left(\varphi+\omega_{1}\right) \sin \left(\varphi+\omega_{2}\right) \sin \left(\varphi+\omega_{3}\right),
\end{aligned}
$$

where $\omega_{1}, \omega_{2}$ and $\omega_{3}$ are real or complex numbers. First integrals are the following. 
(iv.1) If $\omega_{i}-\omega_{j} \neq k \pi / 2, k=0,1, i<j$ and $i, j=1,2,3$, then

$$
\begin{aligned}
H(r, \varphi)= & \left(1+\lambda \cos \left(\omega_{2}-\omega_{3}\right) \sin \left(\varphi+\omega_{1}\right) r\right)^{\tan \left(\omega_{2}-\omega_{3}\right)} \\
& \times \frac{\left(1+\lambda \cos \left(\omega_{1}-\omega_{2}\right) \sin \left(\varphi+\omega_{3}\right) r\right)^{\tan \left(\omega_{1}-\omega_{2}\right)}}{\left(1+\lambda \cos \left(\omega_{1}-\omega_{3}\right) \sin \left(\varphi+\omega_{2}\right) r\right)^{\tan \left(\omega_{1}-\omega_{3}\right)}} .
\end{aligned}
$$

(iv.2) If $\omega_{2}=\omega_{3}-\pi / 2$ then

$$
\begin{aligned}
H(r, \varphi)= & \cot \left(\omega_{1}-\omega_{2}\right) \ln \left(1+\lambda \sin \left(\omega_{1}-\omega_{2}\right) \sin \left(\varphi+\omega_{2}\right) r\right) \\
& +\tan \left(\omega_{1}-\omega_{2}\right) \ln \left(1+\lambda \cos \left(\omega_{1}-\omega_{2}\right) \cos \left(\varphi+\omega_{2}\right) r\right) \\
& -\lambda \sin \left(\varphi+\omega_{1}\right) r .
\end{aligned}
$$

(iv.3) If $\omega_{2}=\omega_{3}$ then

$$
\begin{aligned}
H(r, \varphi)= & \frac{1+\lambda \cos ^{2}\left(\omega_{1}-\omega_{2}\right) \sin \left(\varphi+\omega_{1}\right) r}{1+\lambda \cos \left(\omega_{1}-\omega_{2}\right) \sin \left(\varphi+\omega_{2}\right) r} \\
& +\ln \left(\frac{1+\lambda \cos \left(\omega_{1}-\omega_{2}\right) \sin \left(\varphi+\omega_{2}\right) r}{\left(1+\lambda \sin \left(\varphi+\omega_{1}\right) r\right)^{\cos ^{2}\left(\omega_{1}-\omega_{2}\right)}}\right) .
\end{aligned}
$$

The constants $V_{4}, V_{6}$ and $V_{8}$ have been determined for the first time by Bautin [1]. First integrals in different coordinates were given by Lunkevich and Sibirskiu [5]; later on, Li Chengzhi [3], Sibirskiü [9], Lloyd [4] and Żołądek [10] have given expressions for the constants $V_{4}, V_{6}$ and $V_{8}$ with different criteria. The originality of the theorem in our formulation lies, on the one hand, in the characterization of integrable cases by means of polar coordinates, which is simpler and generalizable, as we have seen in Theorems 1 and 2; on the other hand, in making explicit the first integrals and null divergence factors in all cases.

\section{References}

[1] N. N. Bautin, On the number of limit cycles which appear with the variation of coefficients from an equilibrium position of focus or center type, Mat. Sb. 30 (72) (1952), 181-196 (in Russian); English transl. in Amer. Math. Soc. Transl. 100 (1954).

[2] W. A. Coppel, A survey of quadratic systems, J. Differential Equations 2 (1966), 293-304.

[3] C. Li, Two problems of planar quadratic systems, Sci. Sinica Ser. A 26 (1983), 471-481.

[4] N. G. Lloyd, Small amplitude limit cycles of polynomial differential equations, in: Ordinary Differential Equations and Operators, Lecture Notes in Math. 1032, Springer, 1983, 346-356.

[5] V. A. Lunkevich and K. S. Sibirskiǔ, Integrals of a general quadratic differential system in cases of a center, Differential Equations 18 (1982), 563-568. 
[6] V. V. Nemytskiŭ and V. V. Stepanov, Qualitative Theory of Differential Equations, Princeton University Press, Princeton, N.J., 1960.

[7] S. Shi, A method of constructing cycles without contact around a weak focus, J. Differential Equations 41 (1981), 301-312.

[8] -, On the structure of Poincaré-Lyapunov constants for the weak focus of polynomial vector fields, ibid. 52 (1984), 52-57.

[9] K. S. Sibirskiú, Introduction to the Algebraic Theory of Invariant Differential Equations, Manchester University Press, New York, 1988.

[10] H. Żołądek, On certain generalization of Bautin's Theorem, preprint, Institute of Mathematics, University of Warsaw, 1991.

JAVIER CHAVARRIGA

DEPARTAMENTO DE MATEMATICAS

ESCUELA TÉCNICA SUPERIOR DE INGENIERÍA AGRARIA

UNIVERSIDAD DE LÉRIDA

C/ ROVIRA ROURE, 177

25006 LÉRIDA, SPAIN 